\title{
Analytic Noise Propagation for Anisotropic Denoising of CT Images
}

\author{
Anja Borsdorf ${ }^{1}$, Steffen Kappler ${ }^{2}$, Rainer Raupach ${ }^{2}$, and Joachim Hornegger ${ }^{1}$
}

\begin{abstract}
In X-ray Computed Tomography (CT) the measured projections and consequently the reconstructed CT images are subject to quantum and electronics noise. While noise in the projections can be well described and estimated with a corresponding physics model, the distribution of noise in the reconstructed CT images is not directly evident. Due to attenuation variations along different directions, the nature of noise in CT images is nonstationary and directed. This complicates the direct application of standard post-processing methods like bilateral filtering. In this article we describe a possibility to compute precise orientation dependent noise estimates for every pixel position. This is done by analytic propagation of projection noise estimates through indirect fan-beam filtered backprojection reconstruction. The resulting orientation dependent image noise estimates are subsequently used in adaptive bilateral filters. Taking into account the non-stationary and non-isotropic nature of noise in CT images, an average improvement in SNR of about $60 \%$ is achieved compared to linear filtering at the same resolution.
\end{abstract}

\section{INTRODUCTION}

$\mathbf{T}$ HE non-stationary, object dependent nature of noise in CT images is a direct result of the noise present in the projection data. Since quantum and electronics noise are the dominating noise sources, comparably simple models can be used for direct noise estimates in the individual projections. These noise estimates can be propagated through the reconstruction algorithm in order to get an estimate of the noise variance at every pixel position. In reference [1] we proposed a fast analytic noise propagation through indirect fanbeam filtered-backprojection (FBP) reconstruction. We now extend this noise propagation approach such that separate noise estimates for the horizontal and vertical directions can be computed. This information allows us to adapt filtering methods to the local noise properties in CT images, as we demonstrate by means of a simple bilateral filter [2].

\section{METHOD}

The flowchart of the methodology is presented in Fig. 1 including all intermediate results for an example slice of a real scan. The method splits up into the following parts:

1) The noise variance in the fan-beam projections is estimated according to a calibrated physical noise model.

2) The multiplication with sine and cosine squares separates the contributing rays for noise in horizontal and vertical directions.

${ }^{1}$ A. Borsdorf and J. Hornegger are with University Erlangen-Nuremberg, Chair of Pattern Recognition, Martensstr. 3, 91058 Erlangen, Germany

2 S. Kappler and R. Raupach are with Siemens Healthcare, Siemensstr. 1, 91301 Forchheim, Germany.

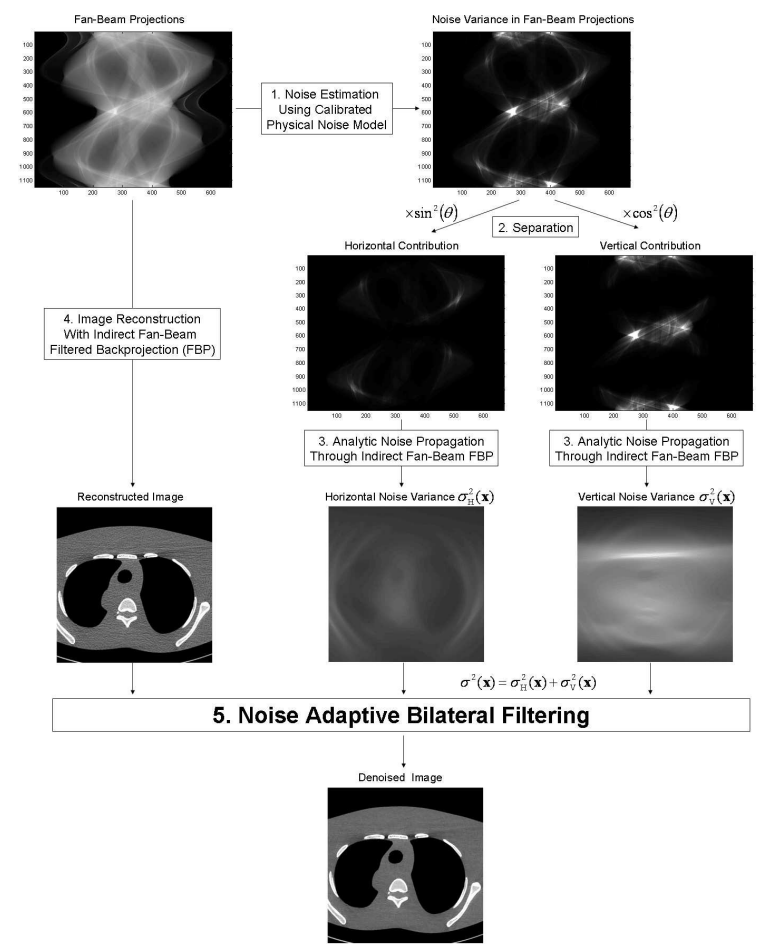

Fig. 1. Flowchart of the noise reduction method.

3) The horizontal and vertical noise variances in image domain are computed using an analytic noise propagation method for indirect fan-beam FBP [1].

4) The CT image is reconstructed using indirect fan-beam FBP reconstruction.

5) The orientation dependent noise estimates in image domain are used for noise adaptive bilateral filtering.

In the following we give a brief review of the noise propagation method and illustrate how it is used for computing orientation dependent noise estimates. After that we describe how these noise estimates are used to adapt the bilateral filter to the non-stationary and non-isotropic noise in the CT image.

\section{A. Orientation Dependent Analytic Noise Propagation}

Our method is based on the analytic propagation of the noise variance through indirect fan-beam reconstruction [1]. Basically, all steps of the reconstruction pipeline (interpolations, convolution and backprojection) can be expressed as 
linear combinations of noisy data (i.e. random variables). For the variance of a linear combination of random variables $X_{i}$ the following holds:

$\operatorname{Var}\left(\sum_{i} a_{i} X_{i}\right)=\sum_{i} a_{i}^{2} \operatorname{Var}\left(X_{i}\right)+2 \sum_{i} \sum_{j \neq i} a_{i} a_{j} \operatorname{Cov}\left(X_{i}, X_{j}\right)$.

Starting from the estimated noise variances in the fan-beam projections $\operatorname{Var}\left(P_{\alpha}^{\mathrm{fan}}(\beta)\right)$ with tube-angle $\alpha$ and fan-angle $\beta$, equation (1) is considered in every single step of the reconstruction algorithm. Further, we model at every step the influence of the processing to the correlation of the data and take this into account for the covariance computation. Finally, we obtain for every pixel position $\mathbf{x}=(x, y)$ of the reconstructed CT image $f(\mathbf{x})$ an estimate of the noise variance $\sigma^{2}(\mathbf{x})$

For objects with strongly directed noise due to strong attenuation along certain directions, one would like to have separate noise estimates for the horizontal and vertical directions. The contribution of noise for every ray of the fan-beam projections to the horizontal direction is computed by:

$$
\operatorname{Var}_{\mathrm{H}}\left(P_{\alpha}^{\mathrm{fan}}(\beta)\right)=\sin ^{2}(\alpha+\beta) \operatorname{Var}\left(P_{\alpha}^{\mathrm{fan}}(\beta)\right),
$$

and analogously for the vertical direction:

$$
\operatorname{Var}_{\mathrm{V}}\left(P_{\alpha}^{\mathrm{fan}}(\beta)\right)=\cos ^{2}(\alpha+\beta) \operatorname{Var}\left(P_{\alpha}^{\mathrm{fan}}(\beta)\right),
$$

where $\theta=\alpha+\beta$ is the parallel projection angle after rebinning. These equations are based on the observation that noise in the projections mostly contributes to the direction orthogonal to the projection direction. The direction dependent noise variances in the projections are then propagated through the reconstruction algorithm as described in [1]. This leads to orientation dependent estimates of the noise standard deviation $\sigma_{\mathrm{H}}(\mathbf{x})$ and $\sigma_{\mathrm{V}}(\mathbf{x})$. A noise estimation vector, defined as:

$$
\bar{\sigma}(\mathbf{x})=\left(\sigma_{\mathrm{H}}(\mathbf{x}), \sigma_{\mathrm{V}}(\mathbf{x})\right),
$$

is now given for every pixel position. Assuming that noise in the fan-beam projections is uncorrelated, the noise variance in the CT image $\sigma^{2}(\mathbf{x})$ can be expressed as:

$$
\sigma^{2}(\mathbf{x})=\sigma_{\mathrm{H}}^{2}(\mathbf{x})+\sigma_{\mathrm{V}}^{2}(\mathbf{x})
$$

This means, that at position $\mathrm{x}$ the standard deviation of noise $\sigma(\mathbf{x})$ is given by the norm of the noise estimation vector defined in eq. (4).

\section{B. Noise-Adaptive Bilateral Filtering}

The orientation dependent noise estimates can be used for denoising the reconstructed CT images with adaptive anisotropic filters. In this article, we restrict to one particular example that is mainly based on the idea of bilateral filtering [2], a simple and widely used approach for edge-preserving denoising. The filtered CT image $\tilde{f}(\mathbf{x})$ is computed as follows:

$$
\tilde{f}(\mathbf{x})=\frac{1}{k(\mathbf{x})} \sum_{\mathbf{x}^{\prime}} f\left(\mathbf{x}^{\prime}\right) \cdot c\left(\mathbf{x}, \mathbf{x}^{\prime}\right) \cdot s\left(f(\mathbf{x}), f\left(\mathbf{x}^{\prime}\right)\right),
$$

where $k(\mathbf{x})$ is needed for normalization. The domain-filter $c\left(\mathbf{x}, \mathbf{x}^{\prime}\right)$ takes into account the geometric closeness of pixels $\mathbf{x}$ and $\mathbf{x}^{\prime}$, and the range-filter $s\left(f(\mathbf{x}), f\left(\mathbf{x}^{\prime}\right)\right)$ considers the photometric closeness of the intensity values during averaging. Instead of using simple Gaussian filters as in [2], we now propose to adapt both parts to the local, orientation dependent noise estimates. We define the domain-filter to be a multivariate Gaussian filter, given by:

$$
\begin{gathered}
c\left(\mathbf{x}, \mathbf{x}^{\prime}\right)=e^{-\frac{1}{2}\left(\left(\mathbf{x}-\mathbf{x}^{\prime}\right)^{T} \Sigma_{\mathbf{x}}^{-1}\left(\mathbf{x}-\mathbf{x}^{\prime}\right)\right)}, \\
\Sigma_{\mathbf{x}}=\left[\begin{array}{cc}
2 \sigma_{\mathrm{H}}^{2}(\mathbf{x}) / \sigma^{2}(\mathbf{x}) & 0 \\
0 & 2 \sigma_{\mathrm{V}}^{2}(\mathbf{x}) / \sigma^{2}(\mathbf{x})
\end{array}\right] d,
\end{gathered}
$$

where $d$ controls the spatial extension of the domain-filter. With increasing $d$ noise is stronger reduced.

For the computation of the range-filter, we now want to take into account the non-stationarity of noise and that directed noise due to strong attenuation along certain directions might be present. We use the noise estimates in horizontal $\sigma_{\mathrm{H}}(\mathbf{x})$ and vertical $\sigma_{\mathrm{V}}(\mathbf{x})$ direction in order to allow stronger filtering to the direction along which less correlation is present. The range-filter is built as a separable filter:

$$
\begin{gathered}
s\left(f(\mathbf{x}), f\left(\mathbf{x}^{\prime}\right)\right)=s_{\mathrm{H}}\left(f(\mathbf{x}), f\left(\mathbf{x}^{\prime}\right)\right) \cdot s_{\mathrm{V}}\left(f(\mathbf{x}), f\left(\mathbf{x}^{\prime}\right)\right), \\
s_{\mathrm{H}}\left(f(\mathbf{x}), f\left(\mathbf{x}^{\prime}\right)\right)=e^{-\frac{1}{2}\left(\frac{\left(\left\|f(\mathbf{x})-f\left(\mathbf{x}^{\prime}\right)\right\| \cos (\varphi)\right)^{2}}{\left(\sigma_{\mathrm{H}}^{2}(\mathbf{x})+\sigma_{\mathrm{H}}^{2}\left(\mathbf{x}^{\prime}\right)\right) s}\right)}, \\
s_{\mathrm{V}}\left(f(\mathbf{x}), f\left(\mathbf{x}^{\prime}\right)\right)=e^{-\frac{1}{2}\left(\frac{\left(\left\|f(\mathbf{x})-f\left(\mathbf{x}^{\prime}\right)\right\| \sin (\varphi)\right)^{2}}{\left(\sigma_{\mathrm{V}}^{2}(\mathbf{x})+\sigma_{\mathrm{V}}^{2}\left(\mathbf{x}^{\prime}\right)\right) s}\right)},
\end{gathered}
$$

where $\varphi$ is the angle between the vector $\left(\mathbf{x}^{\prime}-\mathbf{x}\right)$ and the $x$-axis and the parameter $s$ controls the strength of noise reduction versus edge-preservation. With increasing $s$ more noise is removed.

\section{EXPERIMENTAL EVALUATION}

In Fig. 2 the results are displayed for the simulated phantom used for quantitative evaluation. We used an elliptical water cylinder of $40 \mathrm{~cm} / 20 \mathrm{~cm}$ extension in $\mathrm{x} / \mathrm{y}$-direction with a circular inlay of $3.5 \mathrm{~cm}$ radius and placed $12 \mathrm{~cm}$ off-center. The inlay has a contrast of $100 \mathrm{HU}$ compared to water. We simulated noise-free fan-beam projections with 1160 projections per rotation and 672 channels. We then added quantum noise to the projections and generated 500 noisy realizations. All images were then reconstructed with an indirect fan-beam FBP reconstruction using a standard Shepp-Logan filtering kernel and a field-of-view (FOV) of $40 \mathrm{~cm}$. The noise variance in the projections is directly computed with a quantum noise model and the noise-free fan-beam data. One example image is displayed in Fig. 2 together with filtered versions of it using different configurations of filters:

1) Original noisy image: no filtering is applied. See Fig. 2(a).

2) Gaussian filtering: no range filter is used, just the underlying isotropic Gaussian domain filter influences the result. See Fig. 2(b).

3) Standard bilateral filtering: the range filter used $s$ times the average noise variance within the scanned object as a parameter controlling the range filter. See Fig. 2(c).

4) Adaptive domain filtering: only the domain filter is adapted using the noise estimation vector. The range 


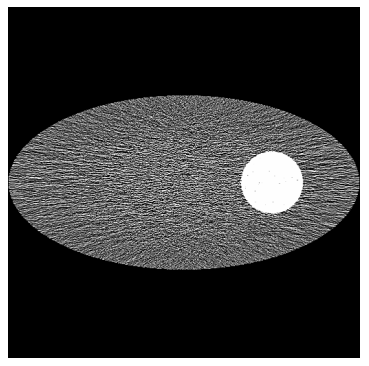

(a) Original noisy image

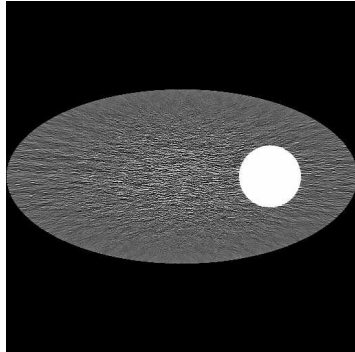

(c) Standard Bilateral Filtering

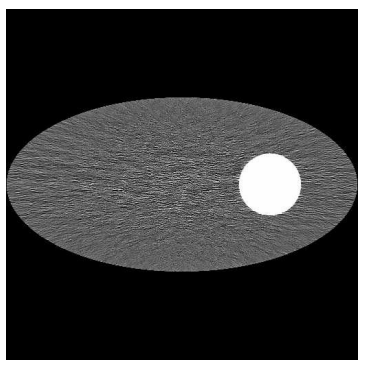

(e) Adaptive Range Filtering

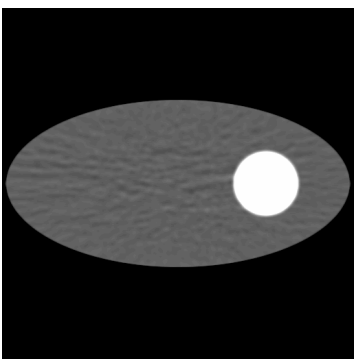

(b) Gaussian

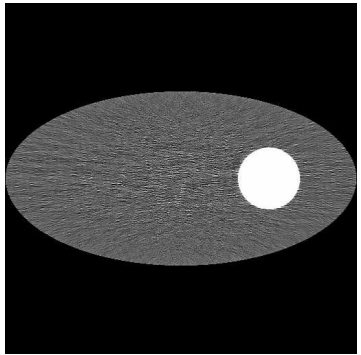

(d) Adaptive Domain Filtering

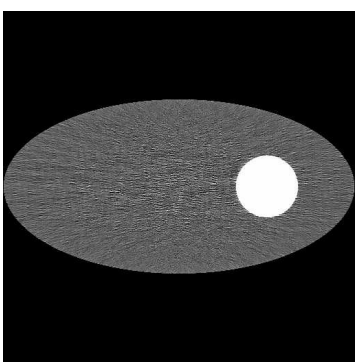

(f) Adaptive Range and Domain Filtering
Fig. 2. Example images used for quantitative evaluation filtered with different configurations of the bilateral filter (all using $\mathrm{d}=9$ and $\mathrm{s}=2$, displayed with $c=10$ and $w=50)$.

filter is just locally adapted by using $s$ times the local noise variance $\sigma^{2}(\mathbf{x})$. See Fig. 2(d).

5) Adaptive range filtering: only the range filter is adapted using the noise estimation vector. The domain filter is an isotropic Gaussian filter. See Fig. 2(e).

6) Adaptive range and domain filtering: both, range and domain filter are adapted using the noise estimation vector. See Fig. 2(f).

Based on the 500 noisy and denoised images, we performed the quantitative evaluation of resolution and noise. For measuring the image resolution we computed the local modulation transfer function (MTF) at the edge of the circular inlay in the average image of the 500 noisy/denoised realizations. The MTF curves for the corresponding configurations listed above are shown in Fig. 3. From the MTF measured at the edge of the processed and original data, the corresponding linear filter was computed that transforms the original MTF into the MTF of the processed data. We then applied this filter as an isotropic linear filter to the noisy images. In average these filtered

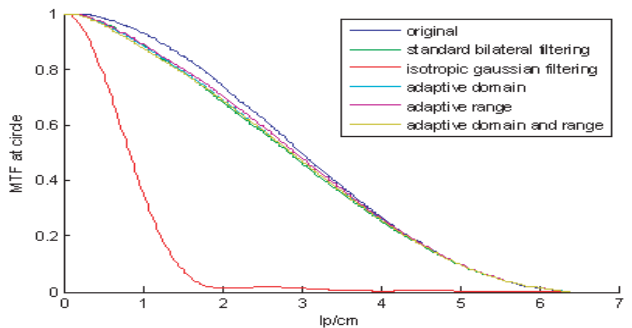

Fig. 3. Local MTFs measured at the circular inlay of $100 \mathrm{HU}$ contrast compared to water.

images have the same average resolution at the circular object, but differ with respect to noise reduction in homogeneous image regions. Therefore, we computed the average standard deviation of noise in the water ellipse in one noise realization of the adaptively filtered and corresponding linearly filtered data. We made sure to leave out the object boundaries in order to only measure the effect of noise reduction. We computed the average standard deviations of noise in the bilateral filtered images, denoted as $\sigma_{\text {filtered }}$ and compared it to the average standard deviations of noise in the same region in the corresponding linearly filtered image $\sigma_{\text {lin.filtered }}$. We then define the gain in signal-to-noise (SNR) ratio as:

$$
\mathrm{SNR}_{\text {gain }}=1-\frac{\sigma_{\text {filtered }}}{\sigma_{\text {lin.filtered }}}
$$

This is a measure of how much more noise can be reduced with the adaptive filtering method compared to a linear filtering leading to the same average resolution at the circular inlay. Therefore, it is a noise-resolution trade-off measurement. The SNR gains achieved for the different configurations are presented in Table I.

TABLE I

SNR GAIN IN COMPARISON TO LINEAR FILTERING WITH SAME SMOOTHING AT THE EDGE FOR THE DIFFERENT FILTERING CONFIGURATIONS

\begin{tabular}{|l||c|c|c|}
\hline configuration & contrast & $\sigma_{\text {filtered }}$ & SNR $_{\text {gain }}$ \\
\hline \hline 1. Original noisy & $100 \mathrm{HU}$ & $16.8 \mathrm{HU}$ & $0 \%$ \\
\hline 2. Gaussian & $100 \mathrm{HU}$ & $0.9 \mathrm{HU}$ & $0 \%$ \\
\hline 3. Standard bilateral & $100 \mathrm{HU}$ & $8.2 \mathrm{HU}$ & $47 \%$ \\
\hline 4. Adaptive domain & $100 \mathrm{HU}$ & $6.5 \mathrm{HU}$ & $58 \%$ \\
\hline 5. Adaptive range & $100 \mathrm{HU}$ & $7.8 \mathrm{HU}$ & $52 \%$ \\
\hline 6. Adaptive range and domain & $100 \mathrm{HU}$ & $6.3 \mathrm{HU}$ & $60 \%$ \\
\hline
\end{tabular}

Furthermore, we compared the standard bilateral filtering and the proposed noise adaptive bilateral filtering approach visually for two different examples of real CT scans. Here we used a calibrated physical noise model for estimating quantum and electronics noise in the fan-beam projections. The images were reconstructed from one single detector row with 672 channels, using 1152 projections per rotation. The FOV is $30 \mathrm{~cm}$ and a standard reconstruction kernel for body scans was used. The original noisy slices together with the two denoised versions are presented in Fig. 4. 


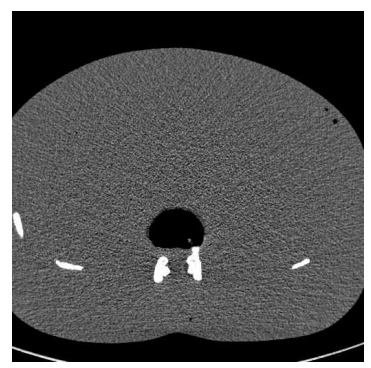

(a) Abdomen Noisy

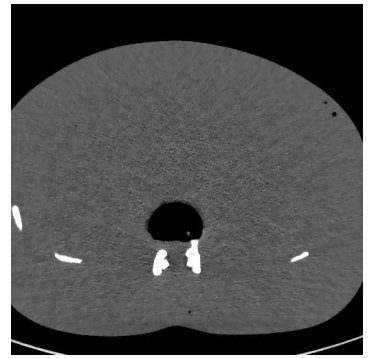

(c) Standard Bilateral Filtering

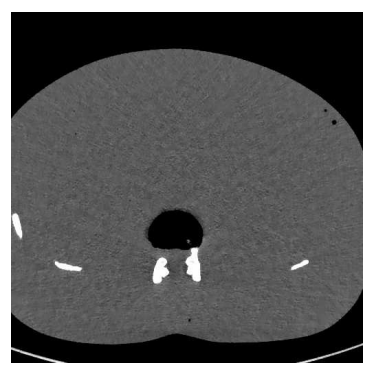

(e) Adaptive Range and Domain Filtering

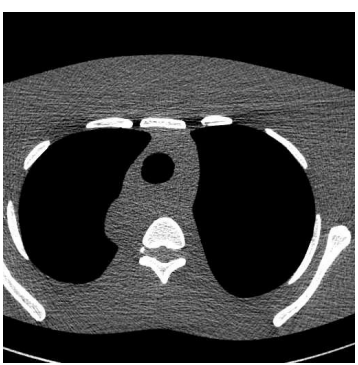

(b) Thorax Noisy

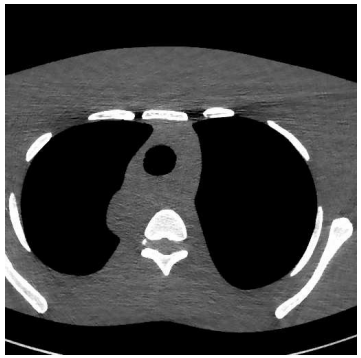

(d) Standard Bilateral Filtering

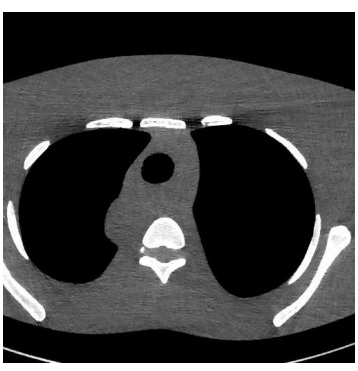

(f) Adaptive Range and Domain Filtering
Fig. 4. Real scanned phantom images from abdomen and thorax region Original noisy images are presented together with standard bilateral filtering and adaptive range and domain filtering results (all using $\mathrm{d}=9$ and $\mathrm{s}=2$, displayed with $c=50$ and $w=400$ ).

\section{DISCUSSION}

The denoising results in Fig. 2 show that with the proposed adaptive bilateral filtering (see Fig. 2(f)) method more homogeneous denoising can be achieved compared to standard bilateral filtering (see Fig. 2(c)).
The proposed method takes into account the local noise variance for controlling the strength of noise reduction. Therefore, the method automatically adapts to the non-stationary noise in the image. Further, the anisotropic adaptation using the horizontal and vertical noise variances allows to reduce strongly directed noise better than the standard filtering method.

The quantitative evaluation based on the simulated data shows that even with standard bilateral filtering edges can be preserved and good noise reduction can be achieved in homogeneous image regions. The proposed method allows the filter to adapt to the non-stationary and non-isotropic noise in CT images. This leads to even better results comparing the noise-resolution-tradeoff measurements presented in Table I. The highest SNR gain of $60 \%$ was achieved for the anisotropic adapted range and domain filter.

These observations are confirmed by the real scanned examples presented in Fig. 4.

\section{CONCLUSIONS}

We proposed a new method for orientation dependent noise estimation in CT images. It is based on analytical propagation of projection noise estimates through indirect fan-beam FBP reconstruction. These noise estimates are then used for adapting bilateral filters to the non-stationary and non-isotropic noise in the CT image. This adaptation leads to improved SNR gain of about $60 \%$ compared to $47 \%$ achieved with standard bilateral filtering at comparable resolution. The horizontal and vertical noise estimates can be used for adapting various other post-processing methods to the very specific noise present in CT images.

\section{ACKNOWLEDGMENT}

The authors gratefully thank Adam Wunderlich, Frédéric Noo, Karl Stierstorfer and Heinrich Wallschläger for helpful suggestions and discussions. Further, the authors acknowledge the financial support by Siemens Healthcare and the International Max-Plack Research School on Optics and Imaging.

\section{REFERENCES}

[1] A. Borsdorf, et al., "Analytic Noise-Propagation in Indirect Fan-Beam FBP Reconstruction," 2008, submitted to IEEE EMBC 2008.

[2] C. Tomasi and R. Manduchi, "Bilateral filtering for gray and color images," in IEEE International Conference on Computer Vision, Bombay, India, 1998, pp. 836-846, http://www.cse.ucsc.edu/ $\sim$ manduchi/Papers/ICCV98.pdf. 